Article

\title{
Comparison of Human Urinary Exosomes Isolated via Ultracentrifugation Alone versus Ultracentrifugation Followed by SEC Column-Purification
}

\author{
Kun Huang ${ }^{1}$, Sudha Garimella ${ }^{2}{ }^{\circledR}$, Alyssa Clay-Gilmour ${ }^{3}$, Lucia Vojtech ${ }^{4}{ }^{\circledR}$, Bridget Armstrong ${ }^{5}$, \\ Madison Bessonny ${ }^{3}$ and Alexis Stamatikos ${ }^{1, *}$
}

1 Department of Food, Nutrition, and Packaging Sciences, Clemson University, Clemson, SC 29634, USA; kunh@g.clemson.edu

2 Prisma Health, Pediatric Nephrology, Greenville, SC 29615, USA; sudha.garimella@prismahealth.org

3 Department of Epidemiology \& Biostatistics, Arnold School of Public Health, University of South Carolina, Columbia, SC 29208, USA; claygila@mailbox.sc.edu (A.C.-G.); bessonny@email.sc.edu (M.B.)

4 Department of Obstetrics \& Gynecology, University of Washington, Seattle, WA 98109, USA; luciav@uw.edu

5 Department of Exercise Science, Arnold School of Public Health, University of South Carolina, Columbia, SC 29208, USA; ba12@mailbox.sc.edu

* Correspondence: adstama@clemson.edu

check for

updates

Citation: Huang, K.; Garimella, S.;

Clay-Gilmour, A.; Vojtech, L.;

Armstrong, B.; Bessonny, M.;

Stamatikos, A. Comparison of

Human Urinary Exosomes Isolated

via Ultracentrifugation Alone versus

Ultracentrifugation Followed by SEC

Column-Purification. J. Pers. Med.

2022, 12, 340. https://doi.org/

10.3390/jpm12030340

Academic Editor: Marijn Speeckaert

Received: 8 February 2022

Accepted: 22 February 2022

Published: 24 February 2022

Publisher's Note: MDPI stays neutral with regard to jurisdictional claims in published maps and institutional affiliations.

Copyright: () 2022 by the authors Licensee MDPI, Basel, Switzerland. This article is an open access article distributed under the terms and conditions of the Creative Commons Attribution (CC BY) license (https:// creativecommons.org/licenses/by/ $4.0 /)$.

\begin{abstract}
Chronic kidney disease is a progressive, incurable condition that involves a gradual loss of kidney function. While there are no non-invasive biomarkers available to determine whether individuals are susceptible to developing chronic kidney disease, small RNAs within urinary exosomes have recently emerged as a potential candidate to use for assessing renal function. Ultracentrifugation is the gold standard for urinary exosome isolation. However, extravesicular small RNA contamination can occur when isolating exosomes from biological fluids using ultracentrifugation, which may lead to misidentifying the presence of certain small RNA species in human urinary exosomes. Therefore, we characterized human urinary exosomal preparations isolated by ultracentrifugation alone, or via ultracentrifugation followed by size exclusion chromatography (SEC) column-purification. Using nanoparticle tracking analysis, we identified SEC fractions containing robust amounts of exosomesized particles, that we further characterized using immunoblotting. When compared to exosomal preparations isolated by ultracentrifugation only, SEC fractionated exosomal preparations showed higher levels of the exosome-positive marker CD81. Moreover, while the exosome-negative marker calnexin was undetectable in SEC fractionated exosomal preparations, we did observe calnexin detection in the exosomal preparations isolated by ultracentrifugation alone, which implies contamination in these preparations. Lastly, we imaged SEC fractionated exosomal preparations using transmission electron microscopy to confirm these preparations contained human urinary exosomes. Our results indicate that combining ultracentrifugation and SEC column-purification exosome isolation strategies is a powerful approach for collecting contaminant-free human urinary exosomes and should be considered when exosomes devoid of contamination are needed for downstream applications.
\end{abstract}

Keywords: biological marker; microRNA; renal failure

\section{Introduction}

Chronic kidney disease (CKD) is a permanent, irreversible condition that involves a gradual loss of kidney function [1]. CKD is thought to afflict millions of Americans annually and many individuals are unaware that they have this condition [2]. There are also no feasible treatments for CKD [1,2]. Patients suffering from CKD may be eligible for kidney transplantation, but some patients who have CKD are at risk of succumbing to this condition before a compatible kidney is available for these individuals due to the lengthy wait time for kidney transplants [3]. There are also many patients afflicted with CKD 
who are deemed ineligible for kidney transplantation [4,5]. Therefore, the predominant treatment for CKD once the kidneys have failed is dialysis [6]. However, dialysis is both burdensome and exhausting on patients, is expensive, and needs to be performed as a regular, life-long therapy [7,8]. Life expectancy and quality-of-life are also drastically decreased in dialysis patients, too [9-11].

While there are several risk factors for CKD, many individuals who develop these risk factors do not acquire CKD [12]. Clinical biomarkers are sometimes used to assess whether a patient may later develop CKD, but some of these biomarkers are collected invasively and have not necessarily been shown to be accurately reliable predictors to determine whether a person may eventually develop CKD [13]. However, data have shown that various dietary and lifestyle patterns may delay or even prevent the onset of CKD [14-16]. Therefore, identifying a simple, economical, and non-invasive biomarker to accurately predict who is prone to developing CKD may dramatically decrease the number of people diagnosed with this condition. Indeed, if individuals are informed that they are susceptible to developing CKD, then they would be much more likely to make dietary/lifestyle changes to reduce the chance of acquiring this disease.

Emerging evidence has shown that the small RNA within human urinary exosomes are promising candidates for assessing kidney function [17-19]. Furthermore, we and others have been successful with detecting microRNA and other small RNA within intact exosomes isolated from various types of biological fluids [20-23]. Urinary collection is easy, inexpensive, and non-invasive, and so there are many advantages to using human urinary exosomal small RNA in a clinical setting. Therefore, if various urinary exosomal microRNA are later determined to be biomarkers for CKD, as well as other diseases, such as cancer or atherosclerosis, then this may greatly assist nephrologists, oncologists, cardiologists, and other types of physicians with diagnosing certain conditions. There are numerous strategies for isolating exosomes from human urine and other biological fluids, with ultracentrifugation being considered the "gold standard" for exosome isolation [24-27]. However, while isolating exosomes via ultracentrifugation is relatively uncomplicated and straightforward, exosomes collected by ultracentrifugation may result in exosomal preparations which contain contamination [28]. A more novel technique for exosome isolation is size exclusion chromatography (SEC) [29], but routine columns used for SEC are unable to accommodate the large urinary volumes needed for exosome isolation. Therefore, we decided to combine ultracentrifugation and SEC column-purification approaches to isolate exosomes from human urine and then compare the isolated exosomes to human urinary exosomes isolated by using ultracentrifugation only. Our results show that while both ultracentrifugation alone and ultracentrifugation followed by SEC column-purification results in collecting robust numbers of human urinary exosomes, only SEC column-purified fractions were shown to be devoid of contamination. Based on these results, we conclude that combining ultracentrifugation and SEC column-purification strategies to isolate exosomes from human urine is superior for obtaining contamination-free exosomal preparations when compared to isolating exosomes from human urine using ultracentrifugation only.

\section{Materials and Methods}

\subsection{Subject Recruitment}

This study was approved by the Institutional Review Board at Prisma Health. We recruited children between the ages of 5-18 years who were attending a primary care clinic. Participants were recruited using fliers and advertisements placed in clinic waiting rooms. Participants were reimbursed $\$ 50$ for completing the assessment. Children were eligible if they had obesity (BMI > 95th \%ile) and were presenting for a well child visit. We selected young age and obesity as inclusion criteria, as childhood and adolescent obesity has been implicated to increase the risk of later developing CKD [30-32]. Exclusion criteria included a diagnosis of hypertension, diabetes, or known kidney or heart disease. Subjects were instructed to come for two study visits 3 months apart. However, for this respective study, a sub-set of samples initially collected during visit 1, was used for analyses. 
Participants $(n=5)$ ranged in age from 7 to 15 (mean age $=11 ; \mathrm{SD}=3.2$ ). Three of the five participants were male. The subjects had an average BMI of 35.1 (SD =11.5). Four of the five participants self-identified their race as Black or African American, and one subject identified as Hispanic/Latinx. Individual level demographics are presented in Table 1.

Table 1. Participant Demographics.

\begin{tabular}{cccccc}
\hline Age & Sex & Race/Ethnicity & Weight & Height & BMI \\
\hline 13 y 0 m & F & Black/African American & 85.5 & 1.627 & 32.30 \\
11 y 6 m & M & Hispanic & 60.1 & 1.478 & 27.51 \\
8 y 3 m & M & Black/African American & 94 & 1.334 & 52.82 \\
7 y 6 m & M & Black/African American & 40.4 & 1.306 & 23.69 \\
15 y 1 m & F & Black/African American & 106 & 1.645 & 39.17 \\
\hline
\end{tabular}

\subsection{Human Urinary Exosome Isolation}

Prior to data collection, informed consent was obtained from primary caregivers and children provided verbal assent. Following the consent procedures, participants completed health questionnaires to assess diet and exercise habits. Anthropometric measurements were collected by trained research staff and BMI was calculated as weight (in kilograms) divided by height (in meters) squared. Participants were mailed sterile urine cups ahead of their visit. They received instructions on how to collect a first morning void and bring it to the visit. Once urine was collected from the human subjects, we immediately transported the urine to Clemson University at $4{ }^{\circ} \mathrm{C}$, so that the urine could be used for human urinary exosome isolation. Briefly, we spun $50 \mathrm{~mL}$ of each participant's voided urine via serial centrifugation at $4{ }^{\circ} \mathrm{C}$, using the following times and speeds per respective spin: (1) $1000 \times g$ for $10 \mathrm{~min}$; (2) $17,000 \times g$ for $15 \mathrm{~min}$; (3) $200,000 \times g$ for $60 \mathrm{~min}$; (4) 200,000 $\times g$ for $60 \mathrm{~min}$. For the first two spins, any pelleted material was discarded, and urine was collected for subsequent spins. For the third spin, exosomes were pelleted, an aliquot of exosome-depleted human urine was collected to use for downstream analysis, and the remaining urine was discarded. The fourth spin consisted of washing the pelleted human urinary exosomes with PBS during this final spin, collecting an aliquot of the PBS wash to use for downstream analysis, removing the remaining PBS wash, and resuspending the washed human urinary exosomes with fresh PBS. Once resuspended, half of the volume of the human urinary exosomal preparations were stored at $-80{ }^{\circ} \mathrm{C}$ to later use for downstream analysis, while the other sample halves were SEC column-purified using Exo-spin $^{\mathrm{TM}}$ mini-HD columns (Cell Guidance Systems LLC, St. Louis, MO, USA) and following manufacturer instructions. Since the manufacturer instructions state fractions 6 and 7 are predicted to have the largest amounts of purified exosomes, we collected both of these fractions, as well as the fractions 5 and 8 because they flanked fractions 6 and 7 and stored all these fractions at $-80{ }^{\circ} \mathrm{C}$, so that they may later be used for downstream analysis.

\subsection{Assessing Human Urinary Particle Size and Number}

Human urinary particle number and diameter size were assessed as previously described [22]. Briefly, human urinary exosomal preparations, exosome-depleted human urine (by ultracentrifugation), and PBS used to wash exosomal preparations during ultracentrifugation were analyzed via nanoparticle tracking analysis (NanoSight NS300 instrument; Malvern Instruments, Malvern, UK) [33]. Human urinary exosomal preparations were initially vortexed and serially diluted with molecular biology grade water and the diluent acted as a negative control. Measurements were taken in replicate fashion, with mean values being calculated.

\subsection{Immunoblotting}

Protein concentrations within human urinary exosomal preparations were measured with a BCA assay kit (BioVision, Milpitas, CA, USA). We separated equal amounts of human urinary exosomal proteins using SDS-PAGE as previously described [34]. These 
separated proteins were subsequently transferred onto PVDF membranes (Merck Millipore Ltd., Burlington, MA, USA) that were incubated in blocking buffer [34] before being probed with the primary antibody mouse anti-CD81 (1:500 dilution, sc-166029; Santa Cruz Biotechnology, Dallas, TX, USA) and primary antibody mouse anti-calnexin (1:1000 dilution, sc-46669; Santa Cruz Biotechnology). We subsequently washed the membranes with TBST after finishing the primary antibody incubation steps and then incubated the membranes with an HRP-conjugated goat anti-mouse IgG secondary antibody (1:5000 dilution, AP181P; Sigma-Aldrich, St. Louis, MO, USA). After the secondary incubation step was complete, we washed membranes with TBST and then incubated the membranes in ECL (Immobilon ECL UltraWestern HRP Substrate; MilliporeSigma, Billerica, MA, USA). We used a ChemiDoc (Analytik Jena US, Upland, CA, USA) for imaging membranes post-ECL incubation [34].

\subsection{Analyzing Human Urinary Exosome Appearance}

To confirm the identity of exosomes within fractionated human urinary exosomal preparations, we imaged the particles in these preparations using transmission electron microscopy [35] and performed this analysis as previously described [22]. Briefly, we pooled together all six and seven human urinary exosomal fractions and added $4 \%$ PFA to this pooled sample. We then deposited the particles mixed with 4\% PFA by airfuge on Formvar-carbon coated transmission electron microscopy grids (Ted Pella, Redding, CA, USA). We then subsequently embedded and contrasted using a uranyl-oxalate solution (pH 7; Electron Microscopy Sciences, Hatfield, PA) for $5 \mathrm{~min}$, and then subsequently performed another treatment using methyl-cellulose-uranyl-acetate (Sigma-Aldrich) on ice for $10 \mathrm{~min}$. Micrograph images were acquired with a JEM-1400 transmission electron microscope (JEOL, Tokyo, Japan) at $120 \mathrm{kV}$ and an Ultrascan 1000XP digital camera (Gatan, Pleasanton, CA, USA).

\section{Results}

3.1. Particle Size Comparisons of Human Urinary Exosomal Preparations Isolated via Ultracentrifugation That Remain Unpurified versus SEC Column-Purified

We used nanoparticle tracking analysis by NanoSight to determine whether the human urinary exosomal preparations we collected using either ultracentrifugation alone or SEC column-purification post-ultracentrifugation contained large numbers of exosome-sized particles. Interestingly, fraction 5 of the SEC column-purified human urinary exosomes was under the limit of detection for NanoSight to accurately measure particle number and diameter size within this fraction. However, fractions 6 and 7 demonstrated a similar diameter size pattern and overall particle number profile when compared to human urinary exosomal preparations isolated via ultracentrifugation only, while we observed a relatively inferior particle number and diameter size in fraction 8 when this fraction was compared to all the other preparations (Figure 1A-E and Supplementary Figure S1A-E). When we performed nanoparticle tracking analysis on exosome-depleted human urine (by ultracentrifugation) and PBS used to wash exosomal preparations during ultracentrifugation and then compared the particle numbers of these control samples to the average particle number of the human urinary exosomes isolated using ultracentrifugation/SEC, there were $>95 \%$ fewer particles detected within the control samples (data not shown). These results imply that SEC column-purification can successfully fractionate human urinary exosomes isolated via ultracentrifugation, resulting in certain fractions being collected that contain robust numbers of exosome-sized particles. 


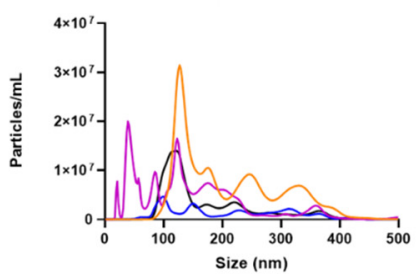

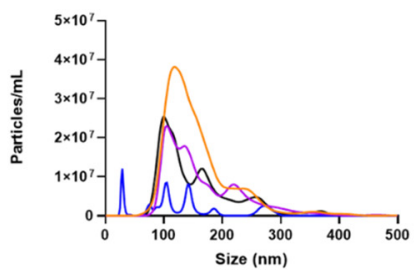

C

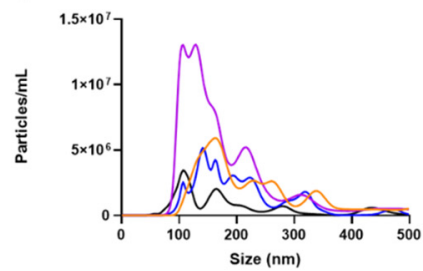

$-F 6$

$-\mathrm{F}^{8}$

- UNp

$-\mathrm{F} 6$
$-\mathrm{F} 7$

- F8

- UNp

D

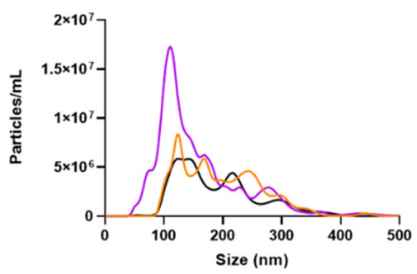

E

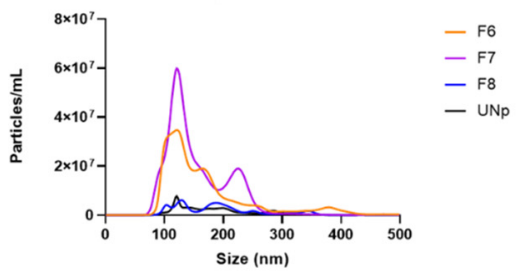

Figure 1. Size distribution of human urinary particles isolated by ultracentrifugation alone versus ultracentrifugation followed by SEC column-purification. (A-E) Human urine was collected from subjects listed in Table 1. Particle diameter size and concentration determined by nanoparticle tracking analysis for human urinary particles isolated using ultracentrifugation and remained unpurified (UNp) or isolated via ultracentrifugation and then fractionated using SEC column-purification, with fraction 6 (F6), fraction 7 (F7), and fraction 8 (F8) being analyzed. A $500 \mathrm{~nm}$ size cutoff was used for data representation. (D) F8 was below the limit of detection.

\subsection{Human Urinary Exosomal Preparations Isolated Using Ultracentrifugation Followed-by SEC Column-Purification Are Devoid of Contamination}

To assess whether our human urinary exosomal preparations are potentially contaminated with non-exosomal proteins, we used immunoblotting to probe for the exosomenegative marker calnexin, which has been identified in human urinary microvesicle/ extracellular vesicle preparations [36]. We also used immunoblotting to probe for the exosome-positive marker and tetraspanin CD81, which has been shown to be present in human urinary exosomes [37]. For immunoblot analyses, we used the human urinary exosomal preparations which had the highest numbers of exosome-sized particles based on nanoparticle tracking analysis, in addition to exosome-depleted human urine (by ultracentrifugation) and PBS used to wash exosomal preparations during ultracentrifugation, which acted as controls. For our results, we detected higher amounts of CD81 protein in fractions 6 and 7 when compared to the human urinary exosomal preparations isolated by ultracentrifugation only, but no protein was detected in the control lanes. However, when we probed for calnexin, this marker was not detected in fractions 6 and 7 or control samples but was detected in some of the human urinary exosomal preparations isolated by ultracentrifugation alone, which suggests contamination is present within the human urinary exosomal preparations that were isolated via ultracentrifugation only (Figure 2A-E).

\subsection{Assessing the Presence of Exosomes in Pooled SEC Fractionated Preparations by Transmission Electron Microscopy}

To confirm the presence of exosomes within preparations collected by SEC columnpurification post-ultracentrifugation, we pooled all fractions 6 and 7 previously used for nanoparticle tracking analysis and immunoblotting to image via transmission electron microscopy. Our imaging analysis identified exosome-sized particles that exhibited distinct exosome characteristics [22] (Figure 3A,B and Supplementary Figure S2A-D), which indicates human urinary exosomes are present in the fractionated preparations that were assessed by transmission electron microscopy. 
A

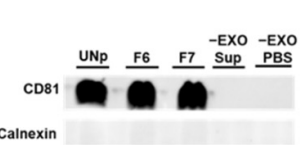

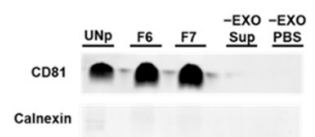

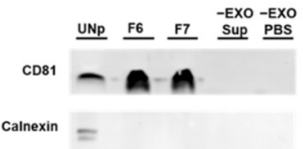

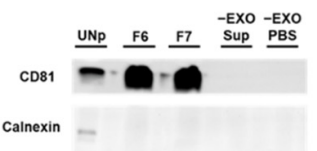

E

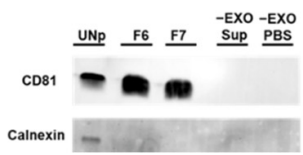

Figure 2. Human urinary exosomal preparations characterized by immunoblotting. (A-E) Human urine was collected from subjects listed in Table 1. Immunoblotting of lysates of human urinary particles isolated using ultracentrifugation and remaining unpurified (UNp) or isolated via ultracentrifugation and then fraction 6 (F6) and fraction 7 (F7) collected by SEC column-purification. Immunoblotting controls were exosome-depleted human urine (-EXO Sup) and PBS used to wash exosomal preparations (-EXO PBS). Blots were probed for the exosome-positive marker CD81 and exosome-negative marker calnexin.

A
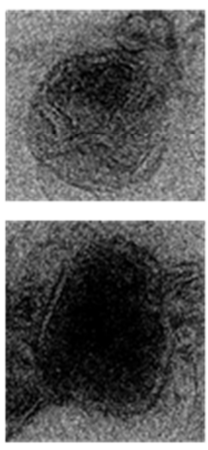

B
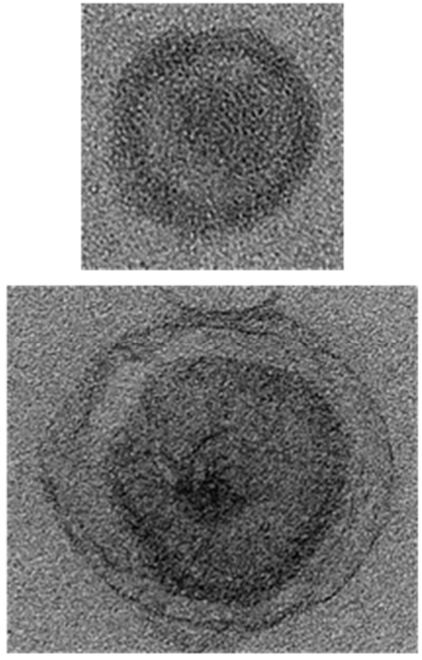

$500 \mathrm{~nm}$

\section{$200 \mathrm{~nm}$}

Figure 3. Pooled human urinary exosomal preparation imaged using transmission electron microscopy. (A,B) Human urinary particles were isolated by ultracentrifugation and further purified using SEC columns. Fraction 6 and fraction 7 were pooled among all collected samples and the pooled preparation was imaged via transmission electron microscopy. The transmission electron micrographs shown are cropped images.

\section{Discussion}

In this study, we wanted to test whether human urinary exosomes which were collected via SEC column-purification post-ultracentrifugation provide preparations that are comparable to human urinary exosomes which were isolated using ultracentrifugation only. In our experiments, we observed fractions 6 and 7 having robust numbers of exosome-sized particles that were similar to the numbers of exosome-sized particles detected in human urinary exosomal preparations isolated by ultracentrifugation alone. However, when we assessed protein levels of the exosome-positive marker CD81, we detected higher amounts of this protein in fractions 6 and 7 when compared to preparations isolated by ultracentrifugation only. Moreover, while we did not detect the exosome-negative marker calnexin in fractions 6 and 7, we did observe this protein being present in some of the preparations which were isolated by ultracentrifugation alone, which implies contamination. We also used transmission electron microscopy to image particles within fractions 6 and 7, which confirmed exosomes were present in these preparations. Therefore, we conclude that isolating human urinary exosomes via ultracentrifugation followed by SEC column-purification 
is superior to ultracentrifugation alone when attempting to collect human urinary exosomal preparations that are free of contamination.

While conventional ultracentrifugation is considered the gold standard for isolating exosomes from biological fluids [24-27], there are several other exosome isolation methods [35]. Over time, there have been newer techniques developed for exosome isolation, as well as attempts to improve older, more traditional exosome isolation methods, including conventional ultracentrifugation $[23,26,27,38]$. For instance, when high-yields of pure exosomal preparations are needed, density gradient ultracentrifugation has been shown to be superior when compared to ultracentrifugation and other exosome isolation techniques $[23,25,39-41]$. Though all downstream applications do not require contaminant-free exosomes, it is noted that these preparations are highly desired when exosomes will later be used for exosomal RNA-seq and next generation sequencing [42-44]. However, density gradient ultracentrifugation is notorious for being difficult and tedious to perform $[23,26,45,46]$. Therefore, a potential alternative strategy to use to effectively isolate high-yields of pure (human urinary) exosomes that is simpler and more convenient than density gradient ultracentrifugation is ultracentrifugation followed by SEC column-purification.

There are two important items we want to emphasize about our study. One, we acknowledge that while our novel exosomal isolation approach which involves combining ultracentrifugation and SEC column-purification appears to work well when isolating exosomes from human urine, caution should be warranted when other types of biological fluid are being used to isolate exosomes. Indeed, it is advised that when SEC fractions are initially collected and originate from a biological fluid that is not human urine, then these fractions should be characterized similarly to what was performed in our respective study, before deciding upon which fractions are most appropriate to use for various downstream applications. While fractions 6 and 7 corresponded with which fractions were predicted to have robust, contaminant-free exosomes based on SEC column manufacturer instructions, it is certainly possible that the many other types of various SEC column-purification methods may result in other fractions containing large amounts of pure exosomes. Therefore, as an initial precaution, it is strongly suggested to analyze all collected fractions by characterization approaches outlined in this study, to better determine which fraction(s) contain large numbers of exosomes that are devoid of contamination.

In conclusion, our results indicate that human urinary exosomes isolated via ultracentrifugation followed by SEC column-purification provide fractions that contain similar numbers of exosomes when compared to human urinary exosomes that are isolated by ultracentrifugation only. However, human urinary exosomes that are isolated via ultracentrifugation alone are shown to have contamination present, while the exosome SEC column-purified fractions do not. Ultimately, for the human urinary exosomes to have any clinical value, a urinary exosomal biomarker or groups of biomarkers that aid in determining whether a patient is at risk of developing CKD, need to be identified. Indeed, if a specific microRNA or cluster of small RNA that is detected within intact human urinary exosomes are shown to increase CKD risk, then these biomarkers could be used clinically to augment other types of renal function tests $[47,48]$. Based on our results, which human urinary exosome isolation method should be utilized may ultimately depend upon whether exosomal preparations need to truly be contaminant-free or not for downstream purposes. If only a few small RNA of interest are attempting to be detected within intact human urinary exosomes, then conventional ultracentrifugation may be a simpler and straightforward method to use for exosome isolation, which can then be followed by an exosome-degradation assay to determine whether the small RNA of interest are actually protected within intact exosomes [22]. However, if researchers want to identify several potential diagnostic biomarkers for CKD (or other conditions) within human urinary exosomes through next generation sequencing, then opting to isolate human urinary exosomes by ultracentrifugation followed by SEC column-purification, would be a more appropriate approach compared to ultracentrifugation only, as the additional SEC step would likely prevent exosomal preparation contamination. 
Supplementary Materials: The following supporting information can be downloaded at: https:// www.mdpi.com/article/10.3390/jpm12030340/s1, Figure S1: Unbiased assessment of human urinary particles isolated by ultracentrifugation alone versus ultracentrifugation coupled with SEC columnpurification.; Figure S2: Unbiased assessment of transmission electron micrographs of a pooled human urinary exosomal preparation.

Author Contributions: Conceptualization, A.S.; methodology, K.H., S.G., A.C.-G., L.V., M.B., B.A. and A.S.; formal analysis, K.H., L.V., B.A. and A.S.; investigation, K.H., S.G., A.C.-G., L.V., M.B., B.A. and A.S.; resources, S.G., A.C.-G., L.V., B.A. and A.S.; writing-original draft preparation, S.G., A.C.-G., B.A. and A.S.; writing-review and editing, S.G., A.C.-G., B.A. and A.S.; supervision, S.G., A.C.-G., B.A. and A.S.; funding acquisition, S.G., A.C.-G. and A.S. All authors have read and agreed to the published version of the manuscript.

Funding: This work was supported in part by a Prisma Health Transformative Seed Grant 2020-2021 and by a USDA National Institute of Food and Agriculture, Hatch project SC-1700577; Accession no. 1021291.

Institutional Review Board Statement: The study was conducted in accordance with the Declaration of Helsinki and approved by the Institutional Review Board of Prisma Health (Pro00097417; Changes in exosomal urinary microRNA expression in obese children as an early biomarker for chronic kidney disease; Initial Approval 4 January 2020).

Informed Consent Statement: Informed consent was obtained from all subjects involved in the study.

Data Availability Statement: All data that is represented within this study is contained in the manuscript.

Conflicts of Interest: The funders had no role in the design of the study; in the collection, analyses, or interpretation of data; in the writing of the manuscript, or in the decision to publish the results.

\section{References}

1. Vaidya, S.R.; Aeddula, N.R. Chronic Renal Failure. In StatPearls; EDN: Treasure Island, FL, USA, 2022.

2. Benjamin, O.; Lappin, S.L. End-Stage Renal Disease. In StatPearls; EDN: Treasure Island, FL, USA, 2022.

3. Davis, A.E.; Mehrotra, S.; McElroy, L.M.; Friedewald, J.J.; Skaro, A.I.; Lapin, B.; Kang, R.; Holl, J.L.; Abecassis, M.M.; Ladner, D.P. The extent and predictors of waiting time geographic disparity in kidney transplantation in the United States. Transplantation 2014, 97, 1049-1057. [CrossRef] [PubMed]

4. Kianda, M.N.; Wissing, K.M.; Broeders, N.E.; Lemy, A.; Ghisdal, L.; Hoang, A.D.; Mikhalski, D.; Donckier, V.; Vereerstraeten, P.; Abramowicz, D. Ineligibility for renal transplantation: Prevalence, causes and survival in a consecutive cohort of 445 patients. Clin. Transplant. 2011, 25, 576-583. [CrossRef] [PubMed]

5. Masaki, N.; Iwadoh, K.; Kondo, A.; Koyama, I.; Nakajima, I.; Fuchinoue, S. Causes of Ineligibility for Recipients in Living Kidney Transplantation. Transplant. Proc. 2018, 50, 978-981. [CrossRef]

6. Chen, T.K.; Knicely, D.H.; Grams, M.E. Chronic Kidney Disease Diagnosis and Management: A Review. JAMA 2019, 322, 1294-1304. [CrossRef] [PubMed]

7. Jameson, M.D.; Wiegmann, T.B. Principles, uses, and complications of hemodialysis. Med. Clin. N. Am. 1990, 74, 945-960. [CrossRef]

8. Vadakedath, S.; Kandi, V. Dialysis: A Review of the Mechanisms Underlying Complications in the Management of Chronic Renal Failure. Cureus 2017, 9, e1603. [CrossRef]

9. Kontodimopoulos, N.; Niakas, D. An estimate of lifelong costs and QALYs in renal replacement therapy based on patients' life expectancy. Health Policy 2008, 86, 85-96. [CrossRef]

10. Md Yusop, N.B.; Yoke Mun, C.; Shariff, Z.M.; Beng Huat, C. Factors associated with quality of life among hemodialysis patients in Malaysia. PLoS ONE 2013, 8, e84152. [CrossRef]

11. Schell, J.O.; Da Silva-Gane, M.; Germain, M.J. Recent insights into life expectancy with and without dialysis. Curr Opin Nephrol Hypertens 2013, 22, 185-192. [CrossRef]

12. Kazancioglu, R. Risk factors for chronic kidney disease: An update. Kidney Int. Suppl. 2013, 3, 368-371. [CrossRef]

13. Lopez-Giacoman, S.; Madero, M. Biomarkers in chronic kidney disease, from kidney function to kidney damage. World J. Nephrol. 2015, 4, 57-73. [CrossRef] [PubMed]

14. Evangelidis, N.; Craig, J.; Bauman, A.; Manera, K.; Saglimbene, V.; Tong, A. Lifestyle behaviour change for preventing the progression of chronic kidney disease: A systematic review. BMJ Open 2019, 9, e031625. [CrossRef] [PubMed]

15. Kramer, H. Diet and Chronic Kidney Disease. Adv. Nutr. 2019, 10 (Suppl. 4), S367-S379. [CrossRef]

16. Michishita, R.; Matsuda, T.; Kawakami, S.; Kiyonaga, A.; Tanaka, H.; Morito, N.; Higaki, Y. The accumulation of healthy lifestyle behaviors prevents the incidence of chronic kidney disease (CKD) in middle-aged and older males. Environ. Health Prev. Med. 2016, 21, 129-137. [CrossRef] [PubMed] 
17. Alvarez, M.L.; Khosroheidari, M.; Kanchi Ravi, R.; DiStefano, J.K. Comparison of protein, microRNA, and mRNA yields using different methods of urinary exosome isolation for the discovery of kidney disease biomarkers. Kidney Int. 2012, 82, 1024-1032. [CrossRef]

18. Khurana, R.; Ranches, G.; Schafferer, S.; Lukasser, M.; Rudnicki, M.; Mayer, G.; Huttenhofer, A. Identification of urinary exosomal noncoding RNAs as novel biomarkers in chronic kidney disease. RNA 2017, 23, 142-152. [CrossRef]

19. Magayr, T.A.; Song, X.; Streets, A.J.; Vergoz, L.; Chang, L.; Valluru, M.K.; Yap, H.L.; Lannoy, M.; Haghighi, A.; Simms, R.J.; et al. Global microRNA profiling in human urinary exosomes reveals novel disease biomarkers and cellular pathways for autosomal dominant polycystic kidney disease. Kidney Int. 2020, 98, 420-435. [CrossRef]

20. Ong, S.G.; Lee, W.H.; Huang, M.; Dey, D.; Kodo, K.; Sanchez-Freire, V.; Gold, J.D.; Wu, J.C. Cross talk of combined gene and cell therapy in ischemic heart disease: Role of exosomal microRNA transfer. Circulation 2014, 130 (Suppl. 1), S60-S69. [CrossRef]

21. Hergenreider, E.; Heydt, S.; Treguer, K.; Boettger, T.; Horrevoets, A.J.; Zeiher, A.M.; Scheffer, M.P.; Frangakis, A.S.; Yin, X.; Mayr, M.; et al. Atheroprotective communication between endothelial cells and smooth muscle cells through miRNAs. Nat. Cell Biol. 2012, 14, 249-256. [CrossRef]

22. Stamatikos, A.; Knight, E.; Vojtech, L.; Bi, L.; Wacker, B.K.; Tang, C.; Dichek, D.A. Exosome-Mediated Transfer of Anti-miR-33a-5p from Transduced Endothelial Cells Enhances Macrophage and Vascular Smooth Muscle Cell Cholesterol Efflux. Hum. Gene Ther. 2020, 31, 219-232. [CrossRef] [PubMed]

23. Van Deun, J.; Mestdagh, P.; Sormunen, R.; Cocquyt, V.; Vermaelen, K.; Vandesompele, J.; Bracke, M.; De Wever, O.; Hendrix, A. The impact of disparate isolation methods for extracellular vesicles on downstream RNA profiling. J. Extracell Vesicles 2014, 3, 24858. [CrossRef] [PubMed]

24. Greening, D.W.; Xu, R.; Ji, H.; Tauro, B.J.; Simpson, R.J. A protocol for exosome isolation and characterization: Evaluation of ultracentrifugation, density-gradient separation, and immunoaffinity capture methods. Methods Mol. Biol. 2015, 1295, 179-209. [PubMed]

25. Lobb, R.J.; Becker, M.; Wen, S.W.; Wong, C.S.; Wiegmans, A.P.; Leimgruber, A.; Moller, A. Optimized exosome isolation protocol for cell culture supernatant and human plasma. J. Extracell Vesicles 2015, 4, 27031. [CrossRef] [PubMed]

26. Yang, D.; Zhang, W.; Zhang, H.; Zhang, F.; Chen, L.; Ma, L.; Larcher, L.M.; Chen, S.; Liu, N.; Zhao, Q.; et al. Progress, opportunity, and perspective on exosome isolation-efforts for efficient exosome-based theranostics. Theranostics 2020, 10, 3684-3707. [CrossRef]

27. Li, P.; Kaslan, M.; Lee, S.H.; Yao, J.; Gao, Z. Progress in Exosome Isolation Techniques. Theranostics 2017, 7, 789-804. [CrossRef]

28. Ludwig, N.; Whiteside, T.L.; Reichert, T.E. Challenges in Exosome Isolation and Analysis in Health and Disease. Int. J. Mol. Sci. 2019, 20, 4684. [CrossRef]

29. Sidhom, K.; Obi, P.O.; Saleem, A. A Review of Exosomal Isolation Methods: Is Size Exclusion Chromatography the Best Option? Int. J. Mol. Sci. 2020, 21, 6466. [CrossRef]

30. Correia-Costa, L.; Azevedo, A.; Caldas Afonso, A. Childhood Obesity and Impact on the Kidney. Nephron 2019, 143, 8-11. [CrossRef]

31. Ding, W.; Cheung, W.W.; Mak, R.H. Impact of obesity on kidney function and blood pressure in children. World J. Nephrol. 2015, 4, 223-229. [CrossRef]

32. Yim, H.E.; Yoo, K.H. Obesity and chronic kidney disease: Prevalence, mechanism, and management. Clin. Exp. Pediatr. 2021, 64, 511-518. [CrossRef]

33. Wright, M. Nanoparticle tracking analysis for the multiparameter characterization and counting of nanoparticle suspensions. Methods Mol. Biol. 2012, 906, 511-524. [PubMed]

34. Huang, K.; Jo, H.; Echesabal-Chen, J.; Stamatikos, A. Combined LXR and RXR Agonist Therapy Increases ABCA1 Protein Expression and Enhances ApoAI-Mediated Cholesterol Efflux in Cultured Endothelial Cells. Metabolites 2021, 11, 640. [CrossRef] [PubMed]

35. Thery, C.; Amigorena, S.; Raposo, G.; Clayton, A. Isolation and characterization of exosomes from cell culture supernatants and biological fluids. Curr. Protoc. Cell Biol. 2006, 30, 3-22. [CrossRef] [PubMed]

36. Perez-Hernandez, J.; Olivares, D.; Forner, M.J.; Ortega, A.; Solaz, E.; Martinez, F.; Chaves, F.J.; Redon, J.; Cortes, R. Urinary exosome miR-146a is a potential marker of albuminuria in essential hypertension. J. Transl. Med. 2018, 16, 228. [CrossRef] [PubMed]

37. Fujita, K.; Kume, H.; Matsuzaki, K.; Kawashima, A.; Ujike, T.; Nagahara, A.; Uemura, M.; Miyagawa, Y.; Tomonaga, T.; Nonomura, N. Proteomic analysis of urinary extracellular vesicles from high Gleason score prostate cancer. Sci. Rep. 2017, 7, 42961. [CrossRef]

38. Chen, J.; Li, P.; Zhang, T.; Xu, Z.; Huang, X.; Wang, R.; Du, L. Review on Strategies and Technologies for Exosome Isolation and Purification. Front. Bioeng. Biotechnol. 2021, 9, 811971. [CrossRef]

39. Brennan, K.; Martin, K.; FitzGerald, S.P.; O'Sullivan, J.; Wu, Y.; Blanco, A.; Richardson, C.; Mc Gee, M.M. A comparison of methods for the isolation and separation of extracellular vesicles from protein and lipid particles in human serum. Sci. Rep. 2020, 10, 1039. [CrossRef]

40. Duong, P.; Chung, A.; Bouchareychas, L.; Raffai, R.L. Cushioned-Density Gradient Ultracentrifugation (C-DGUC) improves the isolation efficiency of extracellular vesicles. PLOS ONE 2019, 14, e0215324. [CrossRef]

41. Li, K.; Wong, D.K.; Hong, K.Y.; Raffai, R.L. Cushioned-Density Gradient Ultracentrifugation (C-DGUC): A Refined and High Performance Method for the Isolation, Characterization, and Use of Exosomes. Methods Mol. Biol. 2018, 1740, 69-83. 
42. Cheng, L.; Sharples, R.A.; Scicluna, B.J.; Hill, A.F. Exosomes provide a protective and enriched source of miRNA for biomarker profiling compared to intracellular and cell-free blood. J. Extracell Vesicles 2014, 3, 23743. [CrossRef]

43. Prendergast, E.N.; de Souza Fonseca, M.A.; Dezem, F.S.; Lester, J.; Karlan, B.Y.; Noushmehr, H.; Lin, X.; Lawrenson, K. Optimizing exosomal RNA isolation for RNA-Seq analyses of archival sera specimens. PLoS ONE 2018, 13, e0196913. [CrossRef] [PubMed]

44. Turchinovich, A.; Drapkina, O.; Tonevitsky, A. Transcriptome of Extracellular Vesicles: State-of-the-Art. Front. Immunol. 2019, 10, 202. [CrossRef] [PubMed]

45. Li, X.; Corbett, A.L.; Taatizadeh, E.; Tasnim, N.; Little, J.P.; Garnis, C.; Daugaard, M.; Guns, E.; Hoorfar, M.; Li, I.T.S. Challenges and opportunities in exosome research-Perspectives from biology, engineering, and cancer therapy. APL Bioeng. $2019,3,011503$. [CrossRef] [PubMed]

46. Inamdar, S.; Nitiyanandan, R.; Rege, K. Emerging applications of exosomes in cancer therapeutics and diagnostics. Bioeng. Transl. Med. 2017, 2, 70-80. [CrossRef]

47. Gounden, V.; Bhatt, H.; Jialal, I. Renal Function Tests. In StatPearls; EDN: Treasure Island, FL, USA, 2022.

48. Stevens, L.A.; Levey, A.S. Measured GFR as a confirmatory test for estimated GFR. J. Am. Soc. Nephrol. 2009, $20,2305-2313$. [CrossRef] 